\title{
Impact of systematics on cosmological parameters from future galaxy cluster surveys
}

\author{
Laura Salvati $^{1,2,3}$, Marian Douspis ${ }^{1}$, and Nabila Aghanim ${ }^{1}$ \\ ${ }^{1}$ Université Paris-Saclay, CNRS, Institut d'Astrophysique Spatiale, 91405 Orsay, France \\ 2 INAF - Osservatorio Astronomico di Trieste, Via G. B. Tiepolo 11, 34143 Trieste, Italy \\ e-mail: laura.salvati@inaf.it \\ 3 IFPU - Institute for Fundamental Physics of the Universe, Via Beirut 2, 34014 Trieste, Italy
}

Received 22 May 2020 / Accepted 24 August 2020

\begin{abstract}
Galaxy clusters are a recent cosmological probe. The precision and accuracy of the cosmological parameters inferred from these objects are affected by the knowledge of cluster physics, entering the analysis through the mass-observable scaling relations, and the theoretical description of their mass and redshift distribution, modelled by the mass function. In this work we forecast the impact of different modelling of these ingredients for clusters detected by future optical and near-IR surveys. We consider the standard cosmological scenario and the case with a time-dependent equation of state for dark energy. We analyse the effect of increasing precision on the scaling relation calibration, finding improved constraints on the cosmological parameters. This higher precision exposes the impact of the mass function evaluation, which is a subdominant source of systematics for current data. We compare two different evaluations for the mass function. In both cosmological scenarios the use of different mass functions leads to biases in the parameter constraints. For the $\Lambda \mathrm{CDM}$ model, we find a $1.6 \sigma$ shift in the $\left(\Omega_{\mathrm{m}}, \sigma_{8}\right)$ parameter plane and a discrepancy of $\sim 7 \sigma$ for the redshift evolution of the scatter of the scaling relations. For the scenario with a time-evolving dark energy equation of state, the assumption of different mass functions results in a $\sim 8 \sigma$ tension in the $w_{0}$ parameter. These results show the impact, and the necessity for a precise modelling, of the interplay between the redshift evolution of the mass function and of the scaling relations in the cosmological analysis of galaxy clusters.
\end{abstract}

Key words. large-scale structure of Universe - galaxies: clusters: general - cosmological parameters

\section{Introduction}

In the cosmological hierarchical scenario, galaxy clusters form in the recent Universe from the collapse of high density fluctuations. The formation and evolution of these objects is strictly related to the growth history of the large scale structure and to the underlying cosmological model. For this reason, in recent years galaxy clusters have emerged as a powerful cosmological probe.

Different wavelength observations provide catalogues of hundreds of objects to be used for the cosmological analysis, such as Planck Collaboration XXIV (2016), de Haan et al. (2016), and Bocquet et al. (2019) at millimetre wavelengths; Abbott et al. (2020) in the optical; and Böhringer et al. (2017) and Pacaud et al. (2018) in X-rays. These different analyses show that the accuracy and precision of the cosmological parameter constraints are affected by systematic uncertainties related to the modelling of different theoretical and observational ingredients.

In general, galaxy cluster number counts are used as a cosmological probe. In the ideal scenario the number counts should coincide with the halo mass function, i.e. the number distribution of clusters in bins of redshift and mass. However, cluster masses cannot be measured directly. It is therefore necessary to rely on observables that act as mass-proxies and that tightly correlate with the underlying cluster mass via some statistical scaling relation. The calibration of these scaling relations (called the mass-calibration problem), represents the current limiting systematic in cluster cosmology studies. Scaling relations are then used, together with a model of the selection process, to transform the theoretical mass function into a prediction for the distribution of clusters in the space of survey observables.

In this scenario the halo mass function itself may be a further source of systematics. The calibration of the mass function is usually obtained through numerical simulations. In recent decades many authors have provided different formulations and calibrations that can be used in the cosmological analysis (see e.g. the discussion in Monaco 2016 and references therein). Nevertheless, it has been shown that the mass function calibration can impact the final results on cosmological parameters up to $\sim 10 \%$ (see e.g. the discussion in Paranjape 2014, Bocquet et al. 2016, 2020). The fitting formulas obtained from numerical simulations may change when different analyses are considered, depending on the initial conditions and assumptions performed during the simulations (e.g. assumed initial cosmology, definition of the cluster mass and detection, resolution of the simulation).

From the cosmological analysis of recently observed cluster samples, the scaling relation calibration stands out as the major source of systematics. However, in the near future different surveys will provide samples of thousands of well-characterised clusters. This large amount of statistics and the availability of multi-wavelength observations will likely improve the precision and accuracy of the calibrations of the scaling relations, reducing the impact on the cosmological parameters. It is time therefore to focus on the other ingredients entering the analysis, for example the mass function. 
In this paper we study the impact of these different systematic sources on the cosmological parameters constrained from galaxy clusters. In particular, we analyse the effect of increasing precision for the scaling relation calibrations and different formulations for the mass function. We build the entire cosmological pipeline and simulate observations from three future surveys: the Euclid telescope (Laureijs et al. 2011), the Large Synoptic Survey Telescope (LSST; LSST Science Collaboration 2009), and the Wide Field Infrared Survey Telescope (WFIRST; Spergel et al. 2015). In this way, we are also able to quantify the impact of different observation strategies, such as the observed area and the covered redshift range.

The paper is structured as follows. In Sect. 2 we describe the method we adopted in the analysis, and we present our results in Sect. 3. We discuss our findings and draw our conclusions in Sects. 4 and 5.

\section{Method}

In this work we study the impact on cosmological constraints inferred from galaxy clusters of systematic effects arising from the uncertainty of the scaling relation calibrations and the choice of halo mass function in the analysis. We consider the galaxy cluster number counts as our observable. We define clusters within the radius $R_{200}$, such that the cluster mean mass overdensity is 200 times the critical density at that redshift, $\rho_{c}(z)$. It implies that the cluster mass is defined as

$M_{200}=\frac{4}{3} \pi R_{200}^{3} \rho_{c}(z)$.

In this section we describe the theoretical model adopted to evaluate the cluster number counts and the experimental characteristics used to simulate mock data. We then describe the fitting procedure, through a Markov chain Monte Carlo analysis.

\subsection{Galaxy cluster number counts}

For the evaluation of galaxy cluster number counts, we follow the analysis in Sartoris et al. (2016). The expected cluster number counts in a given redshift and observed mass $\left(M_{200}^{\mathrm{ob}}\right)$ bin, $N_{\ell, m}$, for a survey with a sky coverage $\Omega_{\text {sky }}$, is defined as

$$
\begin{aligned}
N_{\ell, m}= & \Delta \Omega_{\mathrm{sky}} \int_{z \ell}^{z_{\ell+1}} \mathrm{~d} z \frac{\mathrm{d} V}{\mathrm{~d} z \mathrm{~d} \Omega} \int_{M_{\ell, m}^{\mathrm{ob}}}^{M_{\ell, m+1}^{\mathrm{ob}}} \frac{\mathrm{d} M_{200}^{\mathrm{ob}}}{M_{200}^{\mathrm{ob}}} \\
& \times \int_{0}^{+\infty} \mathrm{d} M \frac{\mathrm{d} n\left(M_{200}, z\right)}{\mathrm{d} M_{200}} p\left(M_{200}^{\mathrm{ob}} \mid M_{200}\right) .
\end{aligned}
$$

In Eq. (2) $d V / d z d \Omega$ is the comoving volume element per unit of redshift and solid angle, $\mathrm{d} n\left(M_{200}, z\right) / \mathrm{d} M_{200}$ is the halo mass function, and $p\left(M_{200}^{\mathrm{ob}} \mid M_{200}\right)$ is the probability of a galaxy cluster with true mass $M$ to have an observed mass $M^{\text {ob }}$. We follow Lima \& Hu (2005) and assume a log-normal probability density, such that

$$
p\left(M_{200}^{\mathrm{ob}} \mid M_{200}\right)=\frac{\exp \left[-x^{2}\left(M_{200}^{\mathrm{ob}}\right)\right]}{\sqrt{2 \pi \sigma_{\ln M_{200}}^{2}}},
$$

with

$$
x\left(M_{200}^{\mathrm{ob}}\right)=\frac{\ln M_{200}^{\mathrm{ob}}-\ln M_{\text {bias }}-\ln M_{200}}{\sqrt{2 \sigma_{\ln M_{200}}^{2}}} .
$$

The combination of Eqs. (2) and (3) provides

$$
\begin{aligned}
N_{\ell, m}= & \frac{\Delta \Omega_{\mathrm{sky}}}{2} \int_{z_{\ell}}^{z_{\ell+1}} \mathrm{~d} z \frac{\mathrm{d} V}{\mathrm{~d} z \mathrm{~d} \Omega} \\
& \times \int_{0}^{+\infty} \mathrm{d} M_{200} \frac{\mathrm{d} n\left(M_{200}, z\right)}{\mathrm{d} M_{200}} \\
& \times\left[\operatorname{erfc}\left(x\left(M_{\ell, m}^{\mathrm{ob}}\right)\right)-\operatorname{erfc}\left(x\left(M_{\ell, m+1}^{\mathrm{ob}}\right)\right)\right],
\end{aligned}
$$

with $\operatorname{erfc}(x)$ being the complementary error function.

The definition of $x\left(M_{200}^{\mathrm{ob}}\right)$ provides the link with the scaling relations, with $\ln M_{\text {bias }}$ being the bias in the mass estimation

$\ln M_{\text {bias }}(z)=B_{M, 0}+\alpha \ln (1+z)$,

and $\sigma_{\ln M}$ the intrinsic scatter in the relation between true and observed mass,

$\sigma_{\ln M}^{2}(z)=\sigma_{\ln M, 0}^{2}-1+(1+z)^{2 \beta}$.

We note that in our analysis we assume the bias for the mass estimation and the intrinsic scatter to be redshift dependent. While these quantities are usually assumed to be constants, it has been shown that a redshift evolution would be necessary in order to provide a more realistic description of the scaling relations (see e.g. Salvati et al. 2019 and references therein).

\subsection{Halo mass function}

In this investigation, we implement two different formulations for the mass function. We compare the results of the analysis from Tinker et al. (2008; hereafter T08) and Despali et al. (2016; hereafter D16), both widely used in the cosmological community. We compare these two formulations since they represent two approaches to evaluating the mass function (see also discussion in Sakr et al. 2018).

The analysis in D16 is based on the original formulation from Sheth \& Tormen (1999) and parametrises the mass function in terms of

$v=\left(\frac{\delta_{c}}{\sigma}\right)^{2}$.

As described in D16, $\delta_{c}$ in Eq. (8) is the critical linear theory overdensity $\delta_{\text {lin }}$ required for spherical collapse, divided by the growth factor, with $\delta_{\text {lin }}$ being

$\delta_{\operatorname{lin}} \simeq \frac{3}{20}(12 \pi)^{2 / 3}[1+0.0123 \log \Omega(z)]$.

The $\sigma$ quantity in Eq. (8) is the standard deviation of density perturbations in a sphere of radius $R=\left(3 M / 4 \pi \rho_{0}\right)^{1 / 3}$, defined in linear regime as

$\sigma^{2}=\frac{1}{2 \pi^{2}} \int \mathrm{d} k k^{2} P(k, z)|W(k R)|^{2}$,

where $W(k R)$ is the window function of a spherical top-hat of radius $R$. The mass function then reads

$\frac{\mathrm{d} n}{\mathrm{~d} M}=v f(v) \frac{2 \rho_{0}}{M} \frac{\mathrm{d} \ln \sigma^{-1}}{\mathrm{~d} M}$,

with

$v f(v)=A\left[1+\left(\frac{1}{a v}\right)^{p}\right]\left(\frac{a v}{2 \pi}\right)^{1 / 2} \exp \left(-\frac{a v}{2}\right)$. 
In order to obtain the coefficients $A, a$, and $p$ at $\Delta_{c}=200$, we follow D16 and adopt the definitions

$$
\begin{aligned}
& a=0.4332 x^{2}+0.2263 x+0.7665, \\
& p=-0.1151 x^{2}+0.2554 x+0.2488, \\
& A=-0.1362 x+0.3292
\end{aligned}
$$

where $x$ is defined as $x=\log \left(\Delta_{c} / \Delta_{\text {vir }}\right)$.

The analysis in T08 formulates the mass function in terms of $\sigma$ (as defined in Eq. (10)). The mass function then reads

$$
\frac{\mathrm{d} n}{\mathrm{~d} M}=f(\sigma) \frac{\rho_{0}}{M} \frac{\mathrm{d} \ln \sigma^{-1}}{\mathrm{~d} M}
$$

with

$f(\sigma)=A(z)\left[\left(\frac{\sigma}{b(z)}\right)^{-a(z)}+1\right] \exp \left(-\frac{c(z)}{\sigma^{2}}\right)$.

The coefficients in Eq. (16) are defined as

$A(z)=A_{0}(1+z)^{-0.14}$,
$a(z)=a_{0}(1+z)^{-0.06}$,
$b(z)=b_{0}(1+z)^{-\alpha}$,

$\log \alpha\left(\Delta_{c}\right)=-\left[\frac{0.75}{\log \left(\Delta_{c} / 75\right)}\right]^{1.2}$,

where $A_{0}, a_{0}$, and $b_{0}$ are evaluated at redshift $z=0$ for $\Delta_{c}=200$.

We chose these two formulations also because they both adopt the spherical overdensity algorithm to identify halos. To conclude, we note that these formulations provide consistent results, within $10 \%$, only in the intermediate mass range and in the redshift range up to $z \leq 1.25$, when considering $\Delta_{c}=200$, as discussed in D16.

\subsection{Characteristics of the forecasted experiments}

In this analysis, we consider galaxy clusters detected through future optical and near-IR galaxy surveys. In order to characterise these surveys and build the mock cluster catalogues, we rely on the observed field of view and the covered redshift range. For the cluster selection, we consider a minimum mass threshold as a function of redshift.

In detail, we provide results mimicking the observational strategy for three future experiments, that will provide galaxy cluster catalogues up to high redshift and low mass. We simulate observations for Euclid (Laureijs et al. 2011; labelled "Euclidlike"), LSST (LSST Science Collaboration 2009; labelled "LSST-like"), and WFIRST (Spergel et al. 2015; labelled "WFIRST-like").

In order to simulate the detected clusters for the Euclidlike and LSST-like surveys, we follow the recipe in Ascaso et al. (2017). In detail, to evaluate the Euclid-like selection function, we follow the "Euclid-optimistic scenario" in Ascaso et al. (2017). In this scenario, we expect to detect clusters with completeness and purity greater than $80 \%$ up to redshift $z<1$ and down to mass $8 \times 10^{13} h^{-1} M_{\odot}$. Moving to higher redshift, the same level of completeness and purity is obtained for a higher mass threshold, $2 \times 10^{14} h^{-1} M_{\odot}$, up to redshift $z \simeq 2$. Furthermore, we assume a sky coverage of $15000 \mathrm{deg}^{2}$ and the redshift range $z=[0.1,1.9]$. The mock dataset is therefore characterised by a median redshift $z_{\text {med }}=0.81$ and a median mass $M_{200, \text { med }}^{\mathrm{ob}}=1.53 \times 10^{14}\left[h^{-1} M_{\odot}\right]$.
For the LSST-like experiment, the analysis in Ascaso et al. (2017) shows a cluster selection with a 5\% higher mass limit with respect to the Euclid-optimistic scenario up to redshift $z<$ 0.7 , increasing up to $2 \times 10^{14} h^{-1} M_{\odot}$ at $z=1.4$. In this case, we consider a sky coverage of $18000 \mathrm{deg}^{2}$ and the redshift range $z=[0.1,1.4]$. The mock dataset is therefore characterised by a median redshift $z_{\text {med }}=0.67$ and a median mass $M_{200, \text { med }}^{\mathrm{ob}}=$ $1.79 \times 10^{14}\left[h^{-1} M_{\odot}\right]$.

For the WFIRST-like experiment we follow the recipe in Gehrels \& Spergel (2015) and consider a sky coverage of $2400 \mathrm{deg}^{2}$, with the redshift range $z=[0.1,2.0]$. We assume a cut in mass for the selection function, $M \geq 10^{14}\left[M_{\odot} h^{-1}\right]$. The mock dataset is characterised by a median redshift $z_{\text {med }}=0.90$ and a median mass $M_{200 \text {,med }}^{\mathrm{ob}}=1.75 \times 10^{14}\left[h^{-1} M_{\odot}\right]$. We report in Fig. 1 the simulated cluster number counts as a function of redshift, $N(z)$, and observed mass, $N\left(M_{200}^{\text {ob }}\right)$, for the three experiments.

We note that when simulating the cluster catalogues, we always adopt the mass function formulation from T08.

\subsection{Analysis}

We adopt a Markov chain Monte Carlo (MCMC) approach in the forecast analysis. As MCMC sampler we use the publicly available package cosmomc (Lewis \& Bridle 2002), which relies on a convergence diagnostic based on Gelman and Rubin statistics. We sample at the same time on the cosmological and scaling relation parameters.

For the cosmological model, we first assume the $\Lambda \mathrm{CDM}$ scenario. We vary the six standard parameters: the baryon and CDM densities, $\Omega_{\mathrm{b}}$ and $\Omega_{\mathrm{c}}$; the ratio of the sound horizon to the angular diameter distance at decoupling $\theta$; the scalar spectral index, $n_{s}$; the overall normalisation of the spectrum, $A_{s}$, at $k=0.05 \mathrm{Mpc}^{-1}$; and reionisation optical depth $\tau$. When presenting the results we focus on the parameters describing the matter distribution in the Universe to which galaxy clusters are more sensitive, i.e. the total matter density, $\Omega_{\mathrm{m}}$, and the standard deviation of density perturbations, defined in Eq. (10), evaluated at radius $R=8 \mathrm{Mpc} h^{-1}, \sigma_{8}$.

We then consider the scenario where the equation of state (hereafter EoS) for dark energy varies with time. We adopt the parametrisation from Chevallier \& Polarski (2001) and Linder (2003):

$w=w_{0}+(1-a) w_{a}$.

We recall that galaxy cluster number counts alone are not able to constrain the entire set of cosmological parameters. For this reason we adopt Gaussian priors from the latest Planck release (Planck Collaboration VI 2020) on the baryon density $\Omega_{b} h^{2}$ and the optical depth $\tau$.

The scaling relation parameters are defined in Eqs. (6) and (7). We recall that the overall calibration of the scaling relations acts as a source of systematic uncertainty, when inferring cosmological constraints from galaxy clusters. An inaccurate and imprecise calibration may produce biased cosmological results. In order to quantify the impact of this calibration, we compare scenarios in which the scaling relation parameters are known to different levels of precision. In particular, we adopt the fiducial values: $B_{M, 0}=0, \alpha=0, \sigma_{\ln M}=0.2$, and $\beta=0.125$, and we assume these parameters to be calibrated to a precision of $1 \%, 5 \%$, and $10 \%$. The adopted values are listed in Table 1.

We compare the results for the three simulated experiments, labelled "Euclid-like", "LSST-like", and "WFIRST-like". Furthermore, we compare the effect of the implementation of two 

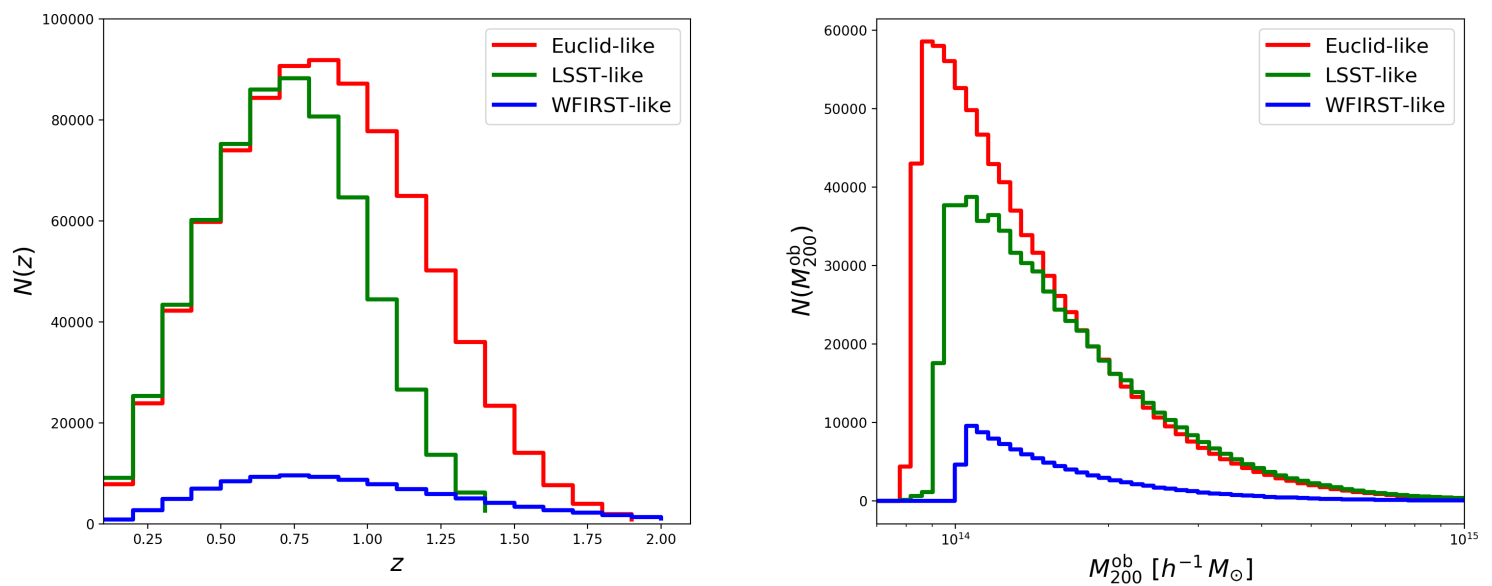

Fig. 1. Simulated cluster number counts as a function of redshift (left) and observed mass (right) for the three experimental set-ups: Euclid-like (red), LSST-like (green), and WFIRST-like (blue).

Table 1. Priors ( $68 \%$ c.l.) on scaling relation parameters applied in the analysis.

\begin{tabular}{cccc}
\hline \hline Parameter & $1 \%$ & $5 \%$ & $10 \%$ \\
\hline$B_{M, 0}$ & $0.0 \pm 0.001$ & $0.0 \pm 0.005$ & $0.0 \pm 0.01$ \\
$\alpha$ & $0.0 \pm 0.002$ & $0.0 \pm 0.01$ & $0.0 \pm 0.02$ \\
$\sigma_{\ln M}$ & $0.2 \pm 0.002$ & $0.2 \pm 0.01$ & $0.2 \pm 0.02$ \\
$\beta$ & $0.125 \pm 0.00125$ & $0.125 \pm 0.00625$ & $0.125 \pm 0.0125$ \\
\hline
\end{tabular}

mass functions T08 and D16 in the pipeline. For the D16 analysis we use as a baseline the case where scaling relation parameters are known at $5 \%$ precision.

\section{Results}

In this section we report our results. We focus on how different precision levels for the scaling relations, formulations for the mass function, and observation strategy affect the estimation of the cosmological parameters. For the last, as discussed in the previous section, we focus on the results for the matter density $\Omega_{\mathrm{m}}$ and the standard deviation of density perturbations $\sigma_{8}$.

We start from the $\Lambda \mathrm{CDM}$ scenario and then discuss an extension of the standard model, with a varying EoS for dark energy.

\section{1. $\Lambda C D M$}

In this section we discuss the effect of the different precision on the scaling relation parameters. We consider three different scenarios where scaling relations parameters are known with a precision of $10 \%, 5 \%$, and $1 \%$. We report the $68 \%$ confidence level (c.l.) constraints for the scaling relation and cosmological parameters in Table 2. In Figs. 2 and 3 we show the $68 \%$ c.l. constraints on the cosmological parameters, for the different precision levels on the scaling relations and for the three experiment configurations. In particular, in Fig. 2 we directly compare the error bars to highlight the impact of the scaling relation precision. As expected, the improvement in the precision leads to an increased constraining power on the cosmological parameters given the degeneracies between these parameters.

In order to discuss how the different experiment characterisations might affect the final results, we chose as a baseline the case where scaling relation parameters are known with a 5\% precision.
We show the comparison between the different experiments, for the cosmological and scaling relation parameters, in the triangular plot in Fig. A.1. On the one hand, the Euclid-like and LSST-like experiments provide tight consistent constraints. As described in Sect. 2.3, the two simulated experiments are indeed characterised by a similar sky coverage while having a different redshift range and selection function. We note that the tighter constraints on the cosmological parameters are also due to the better shaping of the degeneracy with scaling relation parameters. On the other hand, the WFIRST-like experiment provides wider constraints on the cosmological parameters. This experiment is simulated with a lower sky coverage, while spanning up to redshift $z=2$ with a flat selection function. From these results we therefore deduce that the precision on the constraints on cosmological parameters is also affected by the experiment sky coverage.

We conclude this section by discussing the effects of the different evaluations for the mass function. We consider again as the baseline the case with a $5 \%$ precision on the scaling relation parameters and we compare the results obtained using the mass function evaluations from T08 and D16. The 68\% c.l. results for the latter are also reported in Table 2 , and the $68 \%$ c.l. constraints for $\Omega_{\mathrm{m}}$ and $\sigma_{8}$ are shown in Fig. 3. In Fig. 4 we show the two-dimensional probability distributions for $\left(\Omega_{\mathrm{m}}, \sigma_{8}\right)$ for the three simulated experiments, comparing results for the two mass functions.

We recall here that when producing the simulated cluster catalogues, we adopt the T08 formulation for the mass function. When comparing the effect of the mass function formulations in our analysis, on the one hand we confirm that we reproduce the input values for the cosmological parameters when using T08, as expected. On the other hand, the use of D16 introduces biases and shifts in the final results.

We note therefore that the two mass function implementations do not recover the same cosmological parameter constraints. In particular, the impact of the choice of the mass function can be mainly seen on the results from the Euclid-and LSST-like experiments, producing a shift along the $\left(\Omega_{\mathrm{m}}, \sigma_{8}\right)$ degeneracy line up to $1.6 \sigma$. The lower precision of the WFIRSTlike experiment provides larger error bars on the parameter constraints, and therefore does not show the difference between results for the two mass functions: the constraints on $\Omega_{\mathrm{m}}$ and $\sigma_{8}$ are in agreement within $1 \sigma$.

Given the consistency between the Euclid-like and LSSTlike experiments, we focus on the first to further discuss these 
Table 2. Cosmological and scaling relation parameters at the $68 \%$ c.l. in the $\Lambda \mathrm{CDM}$ scenario.

\begin{tabular}{ccccccc}
\hline \hline Experiment & $\Omega_{\mathrm{m}}$ & $\sigma_{8}$ & $B_{M, 0}$ & $\alpha$ & $\sigma_{\ln M}$ & $\beta$ \\
\hline Euclid-like, 1\% SR, T08 & $0.3164 \pm 0.0008$ & $0.8122 \pm 0.0012$ & $0.0 \pm 0.001$ & $0.0 \pm 0.0020$ & $0.2000 \pm 0.0020$ & $0.1250 \pm 0.0011$ \\
Euclid-like, 5\% SR, T08 & $0.3164 \pm 0.0017$ & $0.8119 \pm 0.0033$ & $0.0 \pm 0.005$ & $0.0 \pm 0.010$ & $0.2008 \pm 0.0086$ & $0.1250 \pm 0.0025$ \\
Euclid-like, 5\% SR, D16 & $0.3132 \pm 0.0020$ & $0.8159 \pm 0.0033$ & $0.0063 \pm 0.0048$ & $0.0056 \pm 0.0094$ & $0.2014 \pm 0.0083$ & $0.1056 \pm 0.0025$ \\
Euclid-like, 10\% SR, T08 & $0.3163 \pm 0.0026$ & $0.8115 \pm 0.0049$ & $0.0002 \pm 0.0092$ & $0.001 \pm 0.019$ & $0.203 \pm 0.014$ & $0.1244 \pm 0.0035$ \\
\hline LSST-like, 1\% SR, T08 & $0.3164 \pm 0.0009$ & $0.8122 \pm 0.0013$ & $0.0 \pm 0.001$ & $0.0 \pm 0.002$ & $0.200 \pm 0.002$ & $0.1250 \pm 0.0012$ \\
LSST-like, 5\% SR, T08 & $0.3166 \pm 0.0019$ & $0.8118 \pm 0.0035$ & $0.0 \pm 0.0049$ & $0.0006 \pm 0.0099$ & $0.2006 \pm 0.0086$ & $0.1252 \pm 0.0033$ \\
LSST-like, 5\% SR, D16 & $0.3118 \pm 0.0023$ & $0.8170 \pm 0.0037$ & $0.0027 \pm 0.0049$ & $0.0024 \pm 0.0098$ & $0.1969 \pm 0.0087$ & $0.1099 \pm 0.0034$ \\
LSST-like, 10\% SR, T08 & $0.3166 \pm 0.0030$ & $0.8117 \pm 0.0051$ & $-0.0003 \pm 0.0097$ & $0.002 \pm 0.020$ & $0.202 \pm 0.014$ & $0.1247 \pm 0.0044$ \\
\hline WFIRST-like, 1\% SR, T08 & $0.3168_{-0.0018}^{+0.0016}$ & $0.8116_{-0.0022}^{+0.0020}$ & $0.0 \pm 0.001$ & $0.0 \pm 0.002$ & $0.2001 \pm 0.0019$ & $0.1250 \pm 0.0012$ \\
WFIRST-like, 5\% SR, T08 & $0.3167 \pm 0.0031$ & $0.8120 \pm 0.0050$ & $-0.0002 \pm 0.0050$ & $0.0 \pm 0.01$ & $0.2001 \pm 0.0095$ & $0.1254 \pm 0.0039$ \\
WFIRST-like, 5\% SR, D16 & $0.3184 \pm 0.0035$ & $0.8157 \pm 0.0051$ & $0.0018 \pm 0.0049$ & $0.0 \pm 0.010$ & $0.1960 \pm 0.0095$ & $0.1113 \pm 0.0041$ \\
WFIRST-like, 10\% SR, T08 & $0.3168 \pm 0.0041$ & $0.8118 \pm 0.0076$ & $-0.0003 \pm 0.0098$ & $0.0 \pm 0.02$ & $0.200 \pm 0.018$ & $0.1253 \pm 0.0056$ \\
\hline
\end{tabular}

Notes. We report results for the Euclid-like, LSST-like, and WFIRST-like simulated experiments, for different levels of scaling relation precision and for the T08 and D16 mass function formulations.
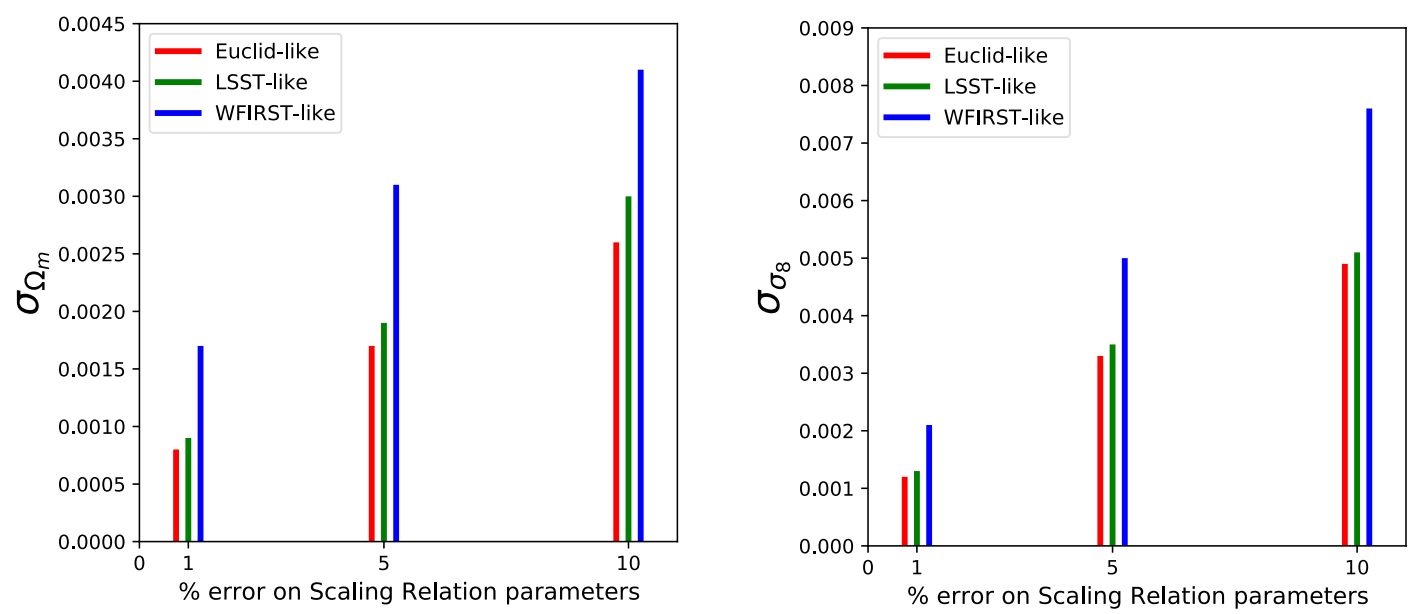

Fig. 2. Error (68\% c.1.) on the cosmological parameters $\Omega_{\mathrm{m}}$ and $\sigma_{8}$ for different levels of precision on the scaling relation parameters $(1 \%, 5 \%$, and $10 \%$ ). Shown are the results for the Euclid-like (red), LSST-like (green), and WFIRST-like (blue) experiments.

results. In the triangular plot in Fig. 5 we show the constraints on cosmological and scaling relation parameters for the Euclidlike mission. From these results we note that the change from the T08 to D16 mass function in the analysis also provides a shift in the scaling relation parameters. In particular, the largest effect can be seen on the $\beta$ parameter. This quantity parametrises the redshift evolution of the scatter of the scaling relations and shows a shift $>7 \sigma$ towards lower values when adopting the D16 evaluation. This shift may represent a general different redshift evolution for the two mass functions that is indeed driving the constraints on $\left(\Omega_{\mathrm{m}}, \sigma_{8}\right)$.

In order to better understand this behaviour, as a further test we check the results when leaving the $\beta$ parameter unconstrained, i.e. not considering the Gaussian prior $\beta=0.125 \pm$ 0.00625 (defined in Table 1). We show the results for $\beta$ and the cosmological parameters $\Omega_{\mathrm{m}}$ and $\sigma_{8}$ in Fig. 6. We note that in this case the parameter shift is even enhanced when considering the D16 mass function, while the results remain consistent for the T08 mass function. We confirm therefore that we are mimicking a different redshift evolution for the two mass functions.

The shift of the scaling relation scatter with respect to redshift implies a change in the total cluster number counts $\mathrm{d} N / \mathrm{d} z$. From previous results (and from discussion in D16), the D16 mass function predicts more clusters at higher $z$. Therefore, the shift in the scaling relation scatter, and in particular having $\beta_{\mathrm{D} 16}<\beta_{\mathrm{T} 08}$, is compensating for this effect.

\section{2. $D E E O S$}

In this section we report the results when varying the EoS for dark energy. We adopt the parametrisation $w=w_{0}+(1-a) w_{a}$.

We follow the same approach as for the standard cosmological scenario and analyse the impact on the cosmological parameter constraints of different precision for the scaling relation parameters, different observation strategies, and mass function implementations.

We list the constraints on the cosmological and scaling relation parameters obtained for the different configurations in Tables 3 and 4 .

In Fig. 7 we give the values of the cosmological parameters with $68 \%$ c.l. error bars, focusing on $w_{0}$ and $w_{a}$. In general, as seen in the previous section, the increasing precision on scaling relation calibration improves the cosmological results. Nevertheless, we note that for the $w_{a}$ parameter the results remain almost unchanged. This may be due to the fact that cluster number counts alone, without the addition of other cosmological probes, 

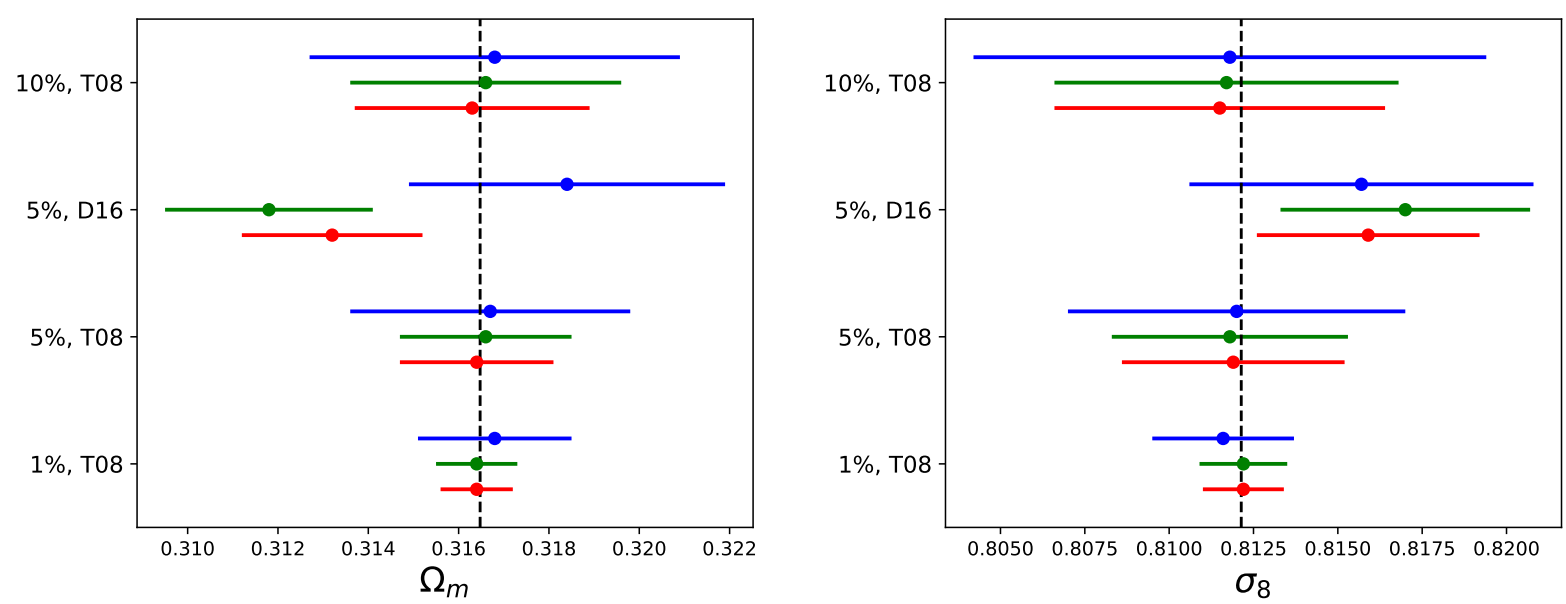

Fig. 3. Values of $\Omega_{\mathrm{m}}$ and $\sigma_{8}$ with $68 \%$ c.l. constraints. Shown are results for the Euclid-like (red), LSST-like (green), and WFIRST-like (blue) experiments, for different levels of scaling relation parameter precision and for the two mass function formulations. The black vertical dashed line represents the input value adopted for the mock data.
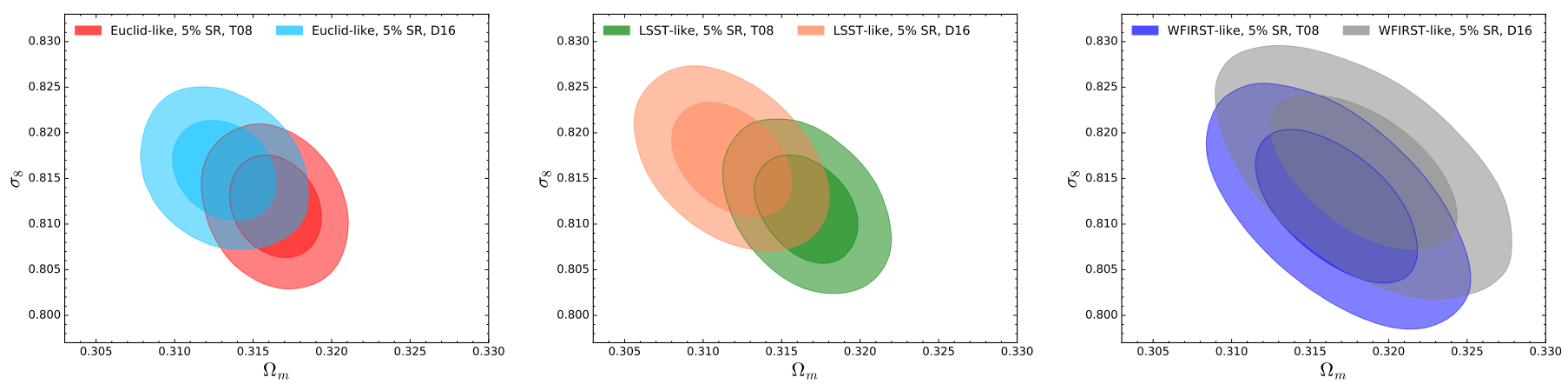

Fig. 4. Two-dimensional probability distributions for $\left(\Omega_{\mathrm{m}}, \sigma_{8}\right)$. Shown are the $68 \%$ and $95 \%$ c.l. marginalised posterior distributions for a $5 \%$ precision on the scaling relation parameters, considering two evaluations of the mass function, T08 and D16. The plots correspond to the Euclidlike survey (left), LSST-like survey (middle), and WFIRST-like survey (right).

are not able to fully constrain the possible redshift evolution of the EoS for dark energy.

As for the $\Lambda$ CDM scenario, the Euclid-like and LSSTlike surveys provide consistent results, while the WFIRST-like experiment produces larger constraints.

We now compare the results when adopting two different mass function formulations. As in the previous section we show and discuss the results for the Euclid-like experiment, assuming a $5 \%$ precision on the scaling relation parameters. In Fig. 8 we show a selection of cosmological and scaling relation parameters results for the T08 (red contours) and the D16 (light blue contours) mass function. On the one hand, in this scenario we note that the constraints on the scaling relation parameters are consistent for the two mass function formulations. We show in Fig. 8 only the results for $\beta$, as a comparison for the shift noted in the $\Lambda \mathrm{CDM}$ scenario.

On the other hand, when adopting D16 mass function, we find a value of the $w_{0}$ parameter that is almost $8 \sigma$ inconsistent with the standard value $w_{0}=-1$. We recall here that changing the dark energy EoS (through a shift in the $w_{0}$ parameter) produces changes to the redshift evolution of the growth factor, and hence to the final cluster number counts. It is possible, therefore, that this shift in $w_{0}$ is again mimicking a different redshift evolution for the two mass functions.

This can be also seen in the results for the matter density $\Omega_{\mathrm{m}}$. When adopting the D16 mass function, the constraints for $\Omega_{\mathrm{m}}$ are shifted towards lower values. This shift compensates for the fact that the D16 mass function predicts higher cluster counts at high redshift.

This trend is also marginally visible in the results for the $\Lambda \mathrm{CDM}$ scenario (as shown in Fig. 5), even though in this case the effect is mainly accounted for in the shift of the $\beta$ parameter.

In order to further check this behaviour and the impact of the redshift evolution for the scatter, we test what happens when forcing $\beta$ to lower values. In particular, we adopt as a prior the constraints obtained for the $\Lambda$ CDM scenario (i.e. $\beta=0.1056 \pm$ $0.0025)$. We report the results in Fig. 8 (grey contours). The lower value of $\beta$ moves the constraints on $w_{0}$ towards higher values, confirming the interplay of these two parameters in describing the redshift dependence on the cluster number counts.

\section{Discussion}

The calibration of the scaling relations, the mass function, and the selection function are crucial issues when dealing with the cosmological analysis of galaxy clusters. A great deal of effort has been focused on this analysis over recent years by the international community.

From currently available cluster catalogues the calibration of scaling relations between cluster mass and survey observables emerges as the main source of systematic uncertainties, while the calibration of the mass function and the selection function provide subdominant impact, of the order of a few percent. 


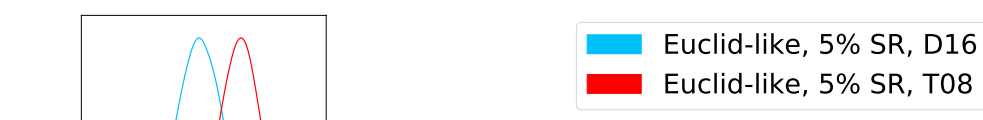

Fig. 5. Constraints on cosmological and scaling relation parameters, in the $\Lambda \mathrm{CDM}$ scenario, for the comparison between the two mass function formulations for the Euclid-like experiment. We show the $68 \%$ and $95 \%$ c.l. marginalised posterior distributions for the 5\% precision on the scaling relation parameters, for the T08 mass function (red) and the D16 mass function (light blue).

The calibration of the scaling relations relies on the tight interplay between cosmology and astrophysics and is usually obtained by exploiting multi-frequency observations. A proper calibration is based on the evaluation of the cluster mass and on the implementation of the relation between this mass and the survey observable.

For the mass evaluation, depending on the frequency range used to detect the clusters, different mass proxies can be considered. For instance, observations at X-ray and millimetre wavelengths target the hot gas in clusters, and therefore make use of properties of the intra-cluster medium as mass proxies. For the observations in the optical regime it is possible to use galaxy kinematics or weak lensing observations. These different methods may provide up to $20 \%$ uncertainties on the mass evaluation. We refer the reader to the extensive discussion in Pratt et al. (2019).

The calibration of the entire relation with the survey observable is usually obtained on a limited number of objects and is then applied to the entire cosmological sample. This approach is based on the assumption that the subsample used for the calibration is actually representative of the total cosmological sample. Furthermore, it requires the understanding of how the total sample maps the underlying population, i.e. a proper description for the selection function. We again refer the reader to the full discussion in Pratt et al. (2019).

Future surveys will provide access to cluster catalogues with $\sim 10^{5}$ elements. This large amount of data will nail down the impact of statistical uncertainties in the cosmological analysis, leaving the results on cosmological constraints to be fully dominated by systematic uncertainties. Therefore, apart from the mass calibration, the full characterisation of the mass function and selection function will be fundamental in order to best exploit the cosmological constraining power of the future surveys.

In this work we focus on the effect of improved precision on the calibration of the scaling relations and the comparison between the two different evaluations of the mass function from 


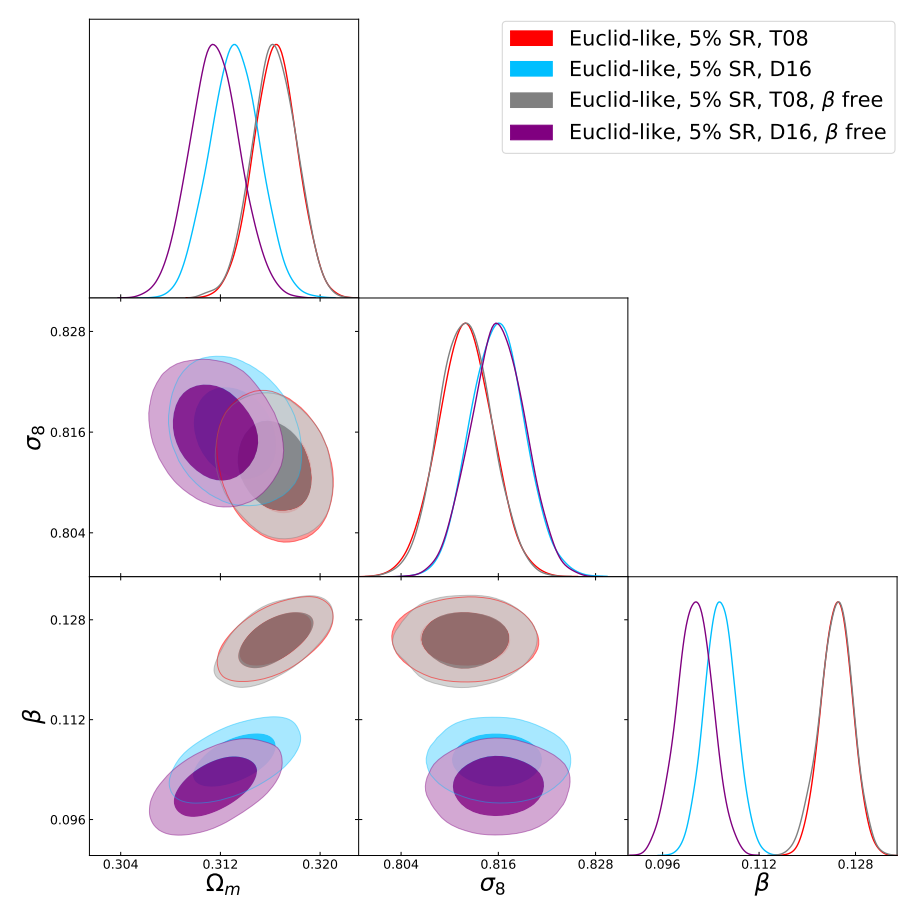

Fig. 6. Constraints on cosmological and $\beta$ parameters for the Euclidlike experiments in the $\Lambda \mathrm{CDM}$ scenario. We assume the scaling relation parameters to be known with a 5\% precision and compare the results for the T08 and D16 mass functions, when considering (red and light blue contours) or not (grey and purple contours) the Gaussian prior on the scaling relation scatter $\beta$. We show the $68 \%$ and $95 \%$ c.l. marginalised posterior distributions.

T08 and D16. Through an MCMC analysis, we forecast how the characterisation of these ingredients impacts the cosmological results from future optical and near-IR galaxy surveys, comparing results for a Euclid-like, an LSST-like, and a WFIRST-like experiment.

In general we find that, on the one hand, increasing the precision on the scaling relation parameters improves the constraining power. On the other hand, the evaluation of the mass function emerges as a dominant source of systematics. We perform this comparative analysis assuming a $5 \%$ precision on the scaling relation parameters. We highlight the following: from the comparison between T08 and D16, we see the interplay of the assumed models for the scaling relations and the mass function in the redshift evolution of the cluster number counts. We model the scaling relations with the mass bias and the scatter to be redshift dependent, through the $\alpha$ and $\beta$ parameters. When analysing the $\Lambda \mathrm{CDM}$ scenario, the comparison between the two mass functions provides consistent results on the cosmological parameters, while we obtain a $7 \sigma$ difference on the $\beta$ parameter. This shift encodes the different redshift evolution of T08 and D16 and in particular the fact that D16 seems to predict more clusters at higher $z$. When considering a varying EoS for dark energy, we find that this different redshift evolution is mimicked by a shift on the matter density $\Omega_{\mathrm{m}}$ and the dark energy parameter $w_{0}$.

From the extensive discussion in D16 we recall here that the two mass function evaluations are consistent within a few percent in the intermediate mass range, while larger differences arise when moving to more massive systems (see also discussion in the Appendix of D16). In this case, the precision of the fit of the mass functions can be strongly affected by the resolution of the simulations used to evaluate the fitting formulas.

Further differences may arise from the choice of the threshold used in the analysis. In our case, we consider galaxy clusters at $\Delta=200 \rho_{c}$, which is shown to provide less agreement between the two formulations. Furthermore, we note that the fitting formula used for D16 has been calibrated in the redshift range up to $z \lesssim 1.25$, while that for T08 up to $z<2.5$. Finally, differences between the two mass functions can be due to the general calibrations that have been adopted, for example from the assumed cosmology in the simulations, from initial conditions, as discussed in Murray et al. (2013), among others.

We note that the impact of the choice of the mass function is different among the three experiments, due to the diverse covered mass and redshift range and distributions, as described in Sect. 2.3. In particular, for the Euclid-like and LSST-like experiments, the different evaluations provide a shift up to $1.6 \sigma$ along the degeneracy line in the $\left(\Omega_{\mathrm{m}}, \sigma_{8}\right)$ plane, apart from the $\sim 7 \sigma$ shift in the $\beta$ parameter. On the contrary, when considering the WFIRST-like experiment, we recover consistent constraints for the cosmological parameters and only a $\sim 3.4 \sigma$ shift in the $\beta$ parameter, in the $\Lambda \mathrm{CDM}$ scenario.

We recall here that in the cosmological analysis of the current cluster samples the scaling relations are calibrated to a precision of $\sim 10-20 \%$, providing uncertainties on the cosmological constraints ranging between $5 \%$ and $20 \%$. As mentioned above, these large errors do not allow us to properly quantify the impact on the cosmological results of the mass function evaluation. Furthermore, we note that the different scaling relation parameters are not known with the same accuracy and precision. These analyses usually encode the redshift dependence for the scaling relations only with the self-similar scenario evolution, not adding for example a redshift dependence for the mass bias or the scatter, as we test in our analysis.

Therefore, we highlight that the precise modelling of the cluster count redshift evolution emerges as a fundamental step to infer cosmological constraints. Given the interplay between scaling relations and mass function, it is necessary to calibrate both on the same cluster sample, spanning a wide range in mass and redshift. This is important especially to reach high accuracy and precision for the determination of the redshift evolution of the scatter and the mass bias.

We conclude by mentioning that in this analysis we did not take into account the impact of the modelling of the selection function, although it represents a fundamental part of the cluster cosmological pipeline. Nevertheless, a proper description of the cluster selection process is strictly related to the final experimental characteristics and scanning strategy. We decided therefore to model it as a redshift-dependent selection in mass, and to focus the analysis on the interconnected impact of the scaling relations and mass function.

\section{Conclusions}

We analysed the impact of the calibration for the scaling relations and the mass function on the cosmological constraints inferred from galaxy clusters detected with future optical and near-IR surveys. We performed the forecast analysis through a Markov chain Monte Carlo approach.

We modelled the experimental set-up for three surveys, spanning different mass and redshift ranges and covering different areas of the sky. We focused on a Euclid-like, an LSST-like, and a WFIRST like survey. In general, the Euclid-like and LSST-like experiments provide consistent results, while the WFIRST-like 
Table 3. Cosmological parameters at the $68 \%$ c.l. when varying the EoS for dark energy.

\begin{tabular}{ccccc}
\hline \hline Experiment & $\Omega_{\mathrm{m}}$ & $\sigma_{8}$ & $w_{0}$ & $w_{a}$ \\
\hline Euclid-like, 1\% SR, T08 & $0.3164 \pm 0.0013$ & $0.8121 \pm 0.0012$ & $-1.0003_{-0.0064}^{+0.0072}$ & $0.12_{-0.18}^{+0.51}$ \\
Euclid-like, 5\% SR, T08 & $0.3165 \pm 0.0018$ & $0.8122 \pm 0.0033$ & $-1.000 \pm 0.010$ & $0.35_{-0.13}^{+0.32}$ \\
Euclid-like, 5\% SR, D16 & $0.3066 \pm 0.0020$ & $0.8153 \pm 0.0034$ & $-1.095 \pm 0.012$ & $0.13_{-0.26}^{+0.51}$ \\
Euclid-like, 10\% SR, T08 & $0.3163 \pm 0.0027$ & $0.8121 \pm 0.0051$ & $-0.999_{-0.011}^{+0.012}$ & $0.28_{-0.20}^{+0.44}$ \\
\hline LSST-like, 1\% SR, T08 & $0.3161_{-0.0016}^{+0.0014}$ & $0.8124_{-0.0013}^{+0.0012}$ & $-1.003 \pm 0.010$ & $0.44_{-0.14}^{+0.47}$ \\
LSST-like, 5\% SR, T08 & $0.3167 \pm 0.0020$ & $0.8121_{-0.0039}^{+0.0034}$ & $-1.000 \pm 0.012$ & $0.33_{-0.21}^{+0.49}$ \\
LSST-like, 10\% SR, T08 & $0.3165 \pm 0.0030$ & $0.8118 \pm 0.0050$ & $-1.000 \pm 0.014$ & $0.17_{-0.20}^{+0.45}$ \\
\hline WFIRST-like, 1\% SR, T08 & $0.3164_{-0.0033}^{+0.0037}$ & $0.8342 \pm 0.0036$ & $-1.000 \pm 0.015$ & $0.33_{-0.22}^{+0.51}$ \\
WFIRST-like, 5\% SR, T08 & $0.3169 \pm 0.0038$ & $0.8119 \pm 0.0050$ & $-0.998 \pm 0.021$ & $0.35_{-0.21}^{+0.46}$ \\
WFIRST-like, 10\% SR, T08 & $0.3167 \pm 0.0044$ & $0.8122 \pm 0.0079$ & $-0.999 \pm 0.024$ & $0.28_{-0.19}^{+0.49}$ \\
\hline
\end{tabular}

Notes. We report the results for the Euclid-like, LSST-like, and WFIRST-like simulated experiments at different levels of scaling relation precision. We show the comparison between the T08 and D16 mass function formulations only for the Euclid-like experiment.

Table 4. Scaling relation parameters at the $68 \%$ c.l. when varying the EoS for dark energy.

\begin{tabular}{ccccc}
\hline \hline Experiment & $B_{M, 0}$ & $\alpha$ & $\sigma_{\ln M}$ & $\beta$ \\
\hline Euclid-like, 1\% SR, T08 & $0.0 \pm 0.001$ & $0.0 \pm 0.0020$ & $0.2000_{-0.0019}^{+0.0018}$ & $0.1250_{-0.0012}^{+0.0011}$ \\
Euclid-like, 5\% SR, T08 & $0.0 \pm 0.005$ & $0.0 \pm 0.010$ & $0.2002 \pm 0.0084$ & $0.1247_{-0.0035}^{+0.0038}$ \\
Euclid-like, 5\% SR, D16 & $-0.0007 \pm 0.0047$ & $0.002 \pm 0.010$ & $0.1935 \pm 0.0088$ & $0.1250 \pm 0.0034$ \\
Euclid-like, 10\% SR, T08 & $0.0 \pm 0.010$ & $0.0 \pm 0.020$ & $0.201 \pm 0.015$ & $0.1245 \pm 0.0051$ \\
\hline LSST-like, 1\% SR, T08 & $0.0 \pm 0.0011$ & $0.0 \pm 0.0019$ & $0.2001 \pm 0.0018$ & $0.1250 \pm 0.0011$ \\
LSST-like, 5\% SR, T08 & $0.0 \pm 0.0047$ & $0.001 \pm 0.010$ & $0.1995 \pm 0.0087$ & $0.1251_{-0.0046}^{+0.0044}$ \\
LSST-like, 10\% SR, T08 & $0.0 \pm 0.010$ & $0.0 \pm 0.019$ & $0.201 \pm 0.015$ & $0.1248 \pm 0.0064$ \\
\hline WFIRST-like, 1\% SR, T08 & $0.0 \pm 0.0010$ & $0.0_{-0.0019}^{+0.0022}$ & $0.1999_{-0.0020}^{+0.0022}$ & $0.1250 \pm 0.0013$ \\
WFIRST-like, 5\% SR, T08 & $0.0 \pm 0.0049$ & $0.0 \pm 0.010$ & $0.200 \pm 0.010$ & $0.1249 \pm 0.0053$ \\
WFIRST-like, 10\% SR, T08 & $0.0 \pm 0.010$ & $0.0 \pm 0.019$ & $0.200 \pm 0.018$ & $0.1249 \pm 0.0077$ \\
\hline
\end{tabular}

Notes. We report results for the Euclid-like, LSST-like, and WFIRST-like simulated experiments at different levels of scaling relation precision. We show the comparison between the T08 and D16 mass function formulations only for the Euclid-like experiment.
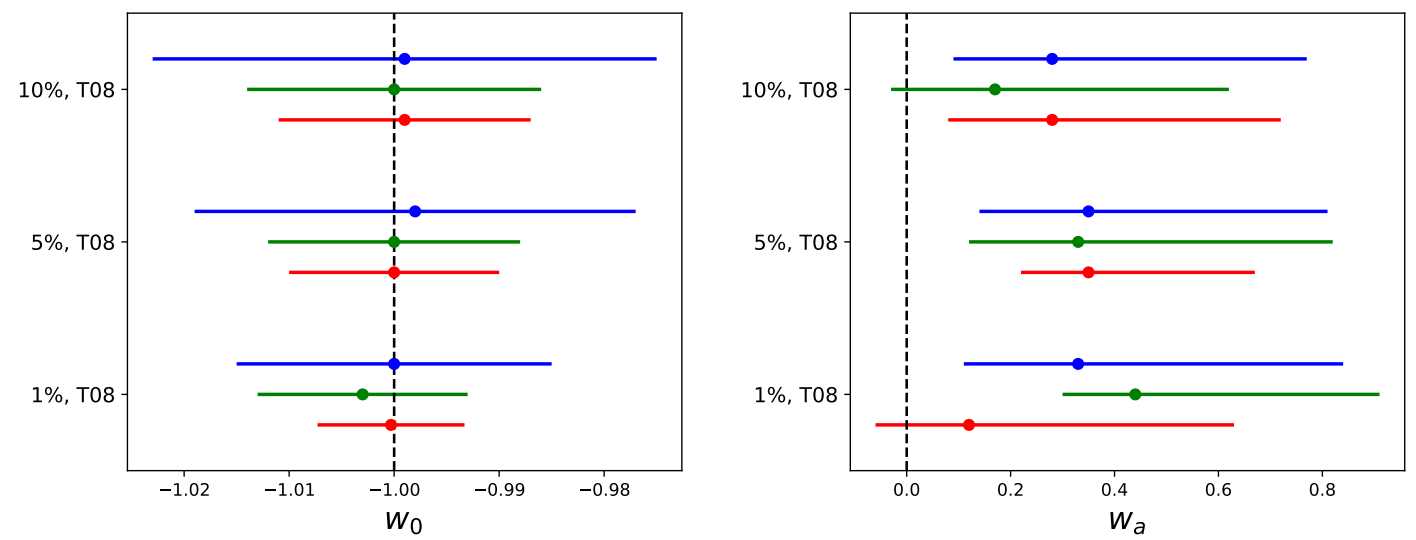

Fig. 7. Values of $w_{0}$ and $w_{a}$ with $68 \%$ c.l. constraints. We report the results for the Euclid-like (red), LSST-like (green), and WFIRST-like (blue) experiments, for different levels of scaling relation parameter precision. The black vertical dashed line represents the input value adopted for the mock data. 


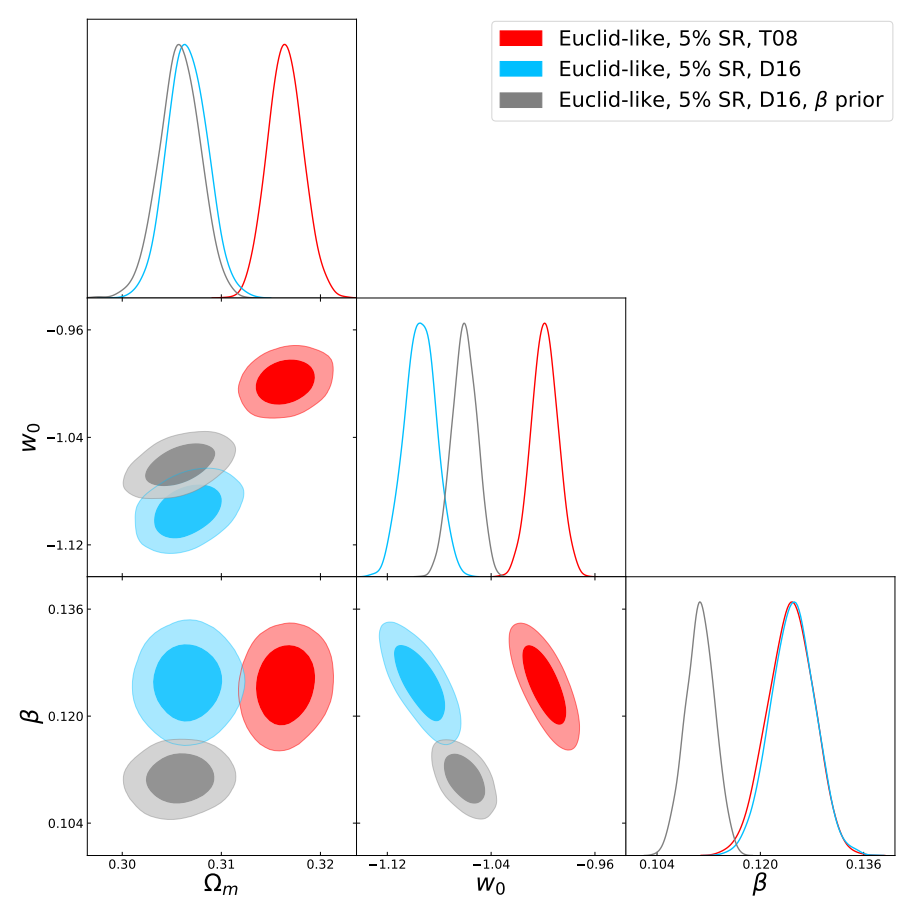

Fig. 8. Constraints on cosmological and scaling relation parameters for the comparison between the two mass function formulations for the Euclid-like experiment. Shown are the results when using the T08 mass function (in red) and when using the D16 mass function (in light blue), and the contours obtained with D16 (in grey) when adopting the prior on the $\beta$ parameter from the $\Lambda \mathrm{CDM}$ analysis. Also sown are the $68 \%$ and $95 \%$ c.l. marginalised posterior distributions

experiment produces larger constraints, mainly due to the substantially smaller observed sky area.

For the scaling relations, we compared results for a $10 \%$, a $5 \%$, and a $1 \%$ precision level on the calibration of the parameters used to describe them. For the mass function, we compared the evaluation from Tinker et al. (2008) and Despali et al. (2016).

We first analysed the impact of these modellings in the $\Lambda \mathrm{CDM}$ scenario. As expected, the increasing precision on the scaling relation parameters provides improved constraints on the cosmological parameters.

The higher precision in the scaling relation calibration exposes the impact of the mass function evaluation, while the latter has only subdominant effects in cluster cosmological analysis from current data. In our analysis, the effect of the two different mass function implementations is mainly seen in the results from the Euclid-like and LSST-like surveys because of their more precise constraints. In particular, the two implemen- tations result in a shift of up to $1.6 \sigma$ in the $\left(\Omega_{\mathrm{m}}, \sigma_{8}\right)$ plane and a discrepancy of $\sim 7 \sigma$ in the redshift dependence for the scatter of the scaling relations. These results might be related to a different redshift evolution of the mass functions.

This hint for a different redshift evolution is confirmed when considering a time-dependent EoS for dark energy, $w=w_{0}+$ $(1-a) w_{a}$. When adopting D16 in the analysis, we find the $w_{0}$ parameter to be in $\sim 8 \sigma$ tension with the standard -1 value. This implies changes in the redshift evolution of the growth factor, and therefore in the final redshift distribution of cluster counts.

We conclude therefore that, apart from the well-known mass calibration problem, a proper evaluation of the mass function emerges as a fundamental issue in the cluster cosmology, especially in view of future large surveys.

Acknowledgements. LS acknowledges support from the postdoctoral grant from Centre National d'Études Spatiales (CNES) and from the ERC-StG "ClustersXCosmo" grant agreement 716762. The authors thank Tiago Batalha de Castro and Stéphane Ilić for useful discussions. This project has received funding from the European Research Council (ERC) under the European Union's Horizon 2020 research and innovation programme grant agreement ERC-2015-AdG 695561 .

\section{References}

Abbott, T., Aguena, M., Alarcon, A., et al. 2020, Phys. Rev. D, 102, 023509 Ascaso, B., Mei, S., Bartlett, J. G., \& Benítez, T. 2017, MNRAS, 464, 2270 Böhringer, H., Chon, G., Retzlaff, J., et al. 2017, AJ, 153, 220

Bocquet, S., Saro, A., Dolag, K., \& Mohr, J. J. 2016, MNRAS, 456, 2361

Bocquet, S., Dietrich, J. P., Schrabback, T., et al. 2019, ApJ, 878, 55

Bocquet, S., Heitmann, K., Habib, S., et al. 2020, ApJ, 901, 5

Chevallier, M., \& Polarski, D. 2001, Int. J. Mod. Phys. D, 10, 213

de Haan, T., Benson, B. A., Bleem, L. E., et al. 2016, ApJ, 832, 95

Despali, G., Giocoli, C., Angulo, R. E., et al. 2016, MNRAS, 456, 2486

Gehrels, N., \& Spergel, D. N. 2015, J. Phys. Conf. Ser., 610, 012007

Laureijs, R., Amiaux, J., Arduini, S., et al. 2011, ArXiv e-prints [arXiv:1110.3193]

Lewis, A., \& Bridle, S. 2002, Phys. Rev. D, 66, 10351

Lima, M., \& Hu, W. 2005, Phys. Rev. D, 72, 043006

Linder, E. V. 2003, Phys. Rev. Lett., 90

LSST Science Collaboration (Abell, P. A., et al.) 2009, ArXiv e-prints [arXiv:0912.0201]

Monaco, P. 2016, Galaxies, 4, 53

Murray, S. G., Power, C., \& Robotham, A. S. G. 2013, MNRAS, 434, L61

Pacaud, F., Pierre, M., Melin, J.-B., et al. 2018, A\&A, 620, A10

Paranjape, A. 2014, Phys. Rev. D, 90, 023520

Planck Collaboration XXIV. 2016, A\&A, 594, A24

Planck Collaboration VI. 2020, A\&A, 641, A6

Pratt, G., Arnaud, M., Biviano, A., et al. 2019, Space Sci. Rev., 215, 25

Sakr, Z., Ilić, S., Blanchard, A., Bittar, J., \& Farah, W. 2018, A\&A, 620, A78

Salvati, L., Douspis, M., Ritz, A., Aghanim, N., \& Babul, A. 2019, A\&A, 626, A27

Sartoris, B., Biviano, A., Fedeli, C., et al. 2016, MNRAS, 459, 1764

Sheth, R. K., \& Tormen, G. 1999, MNRAS, 308, 119

Spergel, D., Gehrels, N., Baltay, C., et al. 2015, ArXiv e-prints [arXiv:1503.03757]

Tinker, J., Kravtsov, A. V., Klypin, A., et al. 2008, ApJ, 688, 709 
L. Salvati et al.: Impact of systematics on cosmological parameters from future galaxy cluster surveys

\section{Appendix A: Results for the LCDM scenario}

We show in the triangular plot in Fig. A.1 the one- and twodimensional probability distributions for the cosmological and scaling relation parameters. We report constraints obtained with a $5 \%$ precision on the scaling relation calibration, comparing results for the Euclid-like, LSST-like, and WFIRST-like experiments.

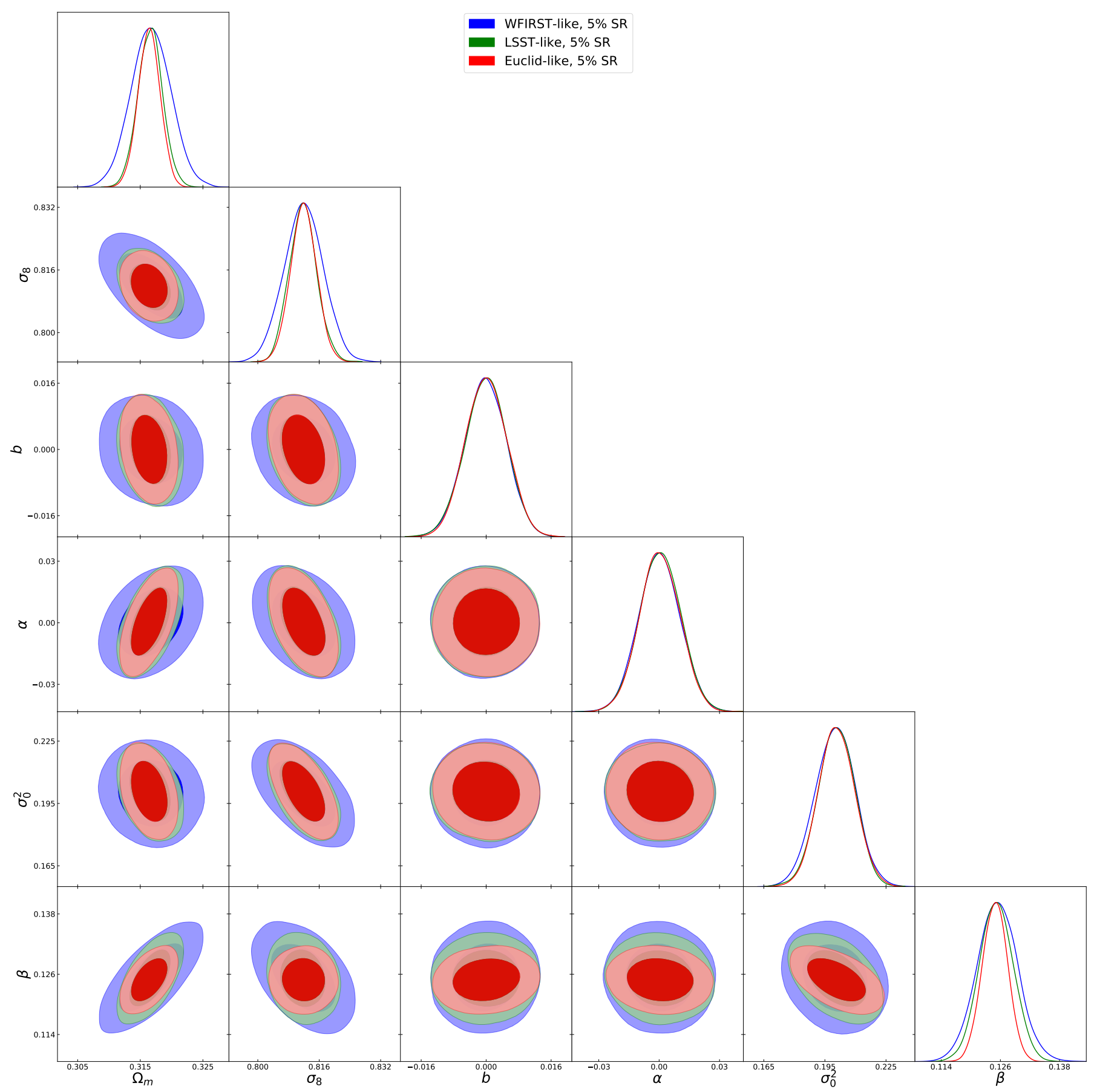

Fig. A.1. Constraints on cosmological and scaling relation parameters for the comparison of the three different experiments: WFIRST-like (blue filled contours), LSST-like (green filled contours), and Euclid-like (red filled contours). Shown are the 68\% and 95\% c.l. marginalised posterior distributions when considering a 5\% error on the scaling relation parameters and the T08 mass function. 